\title{
On the Square Root of a Bell Matrix
}

\author{
Donatella Merlini®
}

\begin{abstract}
In the context of Riordan arrays, the problem of determining the square root of a Bell matrix $R=\mathcal{R}(f(t) / t, f(t))$ defined by a formal power series $f(t)=\sum_{k>0} f_{k} t^{k}$ with $f(0)=f_{0}=0$ is presented. It is proved that if $f^{\prime}(0)=1$ and $f^{\prime \prime}(0) \neq 0$ then there exists another Bell matrix $H=\mathcal{R}(h(t) / t, h(t))$ such that $H * H=R$; in particular, function $h(t)$ is univocally determined by a symbolic computational method which in many situations allows to find the function in closed form. Moreover, it is shown that function $h(t)$ is related to the solution of Schröder's equation. We also compute a Riordan involution related to this kind of matrices.
\end{abstract}

Mathematics Subject Classification. 05A15, 05A19, 05B20, 15A16.

Keywords. Riordan array, Bell matrix, square root matrix, Schröder's equation.

\section{Introduction}

The concept of a (proper) Riordan array was introduced [18,19] as a generalization of the Pascal triangle. A Riordan array is defined as a pair of formal power series $D=\mathcal{R}(d(t), h(t))$ with $d(0) \neq 0, h(0)=0, h^{\prime}(0) \neq 0$. The usual way to represent the Riordan array $\mathcal{R}(d(t), h(t))$ is by means of an infinite matrix $\left(d_{n, k}\right), n, k \in N$, its generic element being:

$$
d_{n, k}=\left[t^{n}\right] d(t) h(t)^{k} .
$$

The Pascal triangle is just the case:

$$
P=\mathcal{R}\left(\frac{1}{1-t}, \frac{t}{1-t}\right) .
$$

Many properties of Riordan arrays have been studied in the literature, in particular their connection with combinatorial sums. Actually, if $\left(s_{n}\right)_{n \in N}$ is 
any sequence having $s(t)=\sum_{k=0}^{\infty} s_{k} t^{k}$ as its generating function, it is possible to prove that:

$$
\sum_{k=0}^{n} d_{n, k} s_{k}=\left[t^{n}\right] d(t) s(h(t))
$$

thus reducing the sum to the extraction of a coefficient from a formal power series.

Riordan arrays constitute a group with respect to the product defined by:

$$
\begin{aligned}
D_{1} * D_{2} & =\mathcal{R}\left(d_{1}(t), h_{1}(t)\right) * \mathcal{R}\left(d_{2}(t), h_{2}(t)\right) \\
& =\mathcal{R}\left(d_{1}(t) d_{2}\left(h_{1}(t)\right), h_{2}\left(h_{1}(t)\right)\right)=\mathcal{R}\left(d_{3}(t), h_{3}(t)\right)=D_{3},
\end{aligned}
$$

which corresponds to the usual row-by-column product of two (infinite) matrices. The Riordan array $I=\mathcal{R}(1, t)$ acts as the identity and the inverse of $D=\mathcal{R}(d(t), h(t))$ is the Riordan array:

$$
D^{*}=\left(d_{n, k}^{*}\right)=\mathcal{R}\left(d^{*}(t), h^{*}(t)\right)=\mathcal{R}\left(\frac{1}{d(\bar{h}(t))}, \quad \bar{h}(t)\right)
$$

where $\bar{h}(t)$ is the compositional inverse of $h(t)$, that is the power series such that $h(\bar{h}(t))=\bar{h}(h(t))=t$. For Pascal triangle we can easily find:

$$
P^{*}=\mathcal{R}\left(\frac{1}{1+t}, \frac{t}{1+t}\right) .
$$

Riordan arrays have another important characterization, due to Rogers [15]: every element $d_{n+1, k+1}, n, k \in N$, can be expressed as a linear combination of the elements in the preceding row, i.e.:

$$
d_{n+1, k+1}=a_{0} d_{n, k}+a_{1} d_{n, k+1}+a_{2} d_{n, k+2}+\cdots=\sum_{j=0}^{\infty} a_{j} d_{n, k+j} .
$$

The sum is actually finite and the sequence $A=\left(a_{k}\right)_{k \in N}$ is called the $A$ sequence of the Riordan array and we have

$$
h(t)=t A(h(t)), \quad \text { or } \quad A(t)=\frac{t}{\bar{h}(t)} .
$$

For example, in the Pascal case we have $A(t)=1+t$. Sometimes, the $A$ sequence is simpler than $h(t)$ and in this case, going through this sequence can simplify the manipulation of the matrix. While computing the product $D_{1} *$ $D_{2}=D_{3}$ between Riordan arrays, He and Sprugnoli [6] proved the following relation involving the corresponding $A$-sequences $A_{1}(t), A_{2}(t)$ and $A_{3}(t)$ :

$$
A_{3}(t)=A_{2}(t) A_{1}\left(\frac{t}{A_{2}(t)}\right) .
$$

There are several known subgroups of the Riordan group, in particular Bell subgroup contains elements of the form $\mathcal{R}(h(t) / t, h(t))$; therefore, a Bell matrix is completely defined by its $A$-sequence. 
In this paper we investigate some questions about powers of Bell matrices. Let us start by considering the generalized Pascal triangle:

$$
P_{r}=\mathcal{R}\left(\frac{1}{1-r t}, \frac{t}{1-r t}\right)
$$

By using the rules for the product between Riordan arrays we can easily compute:

$$
\begin{array}{r}
P_{r} * P_{r}=P_{r}^{2}=\mathcal{R}\left(\frac{1}{1-2 r t}, \frac{t}{1-2 r t}\right) \\
P_{r} * P_{r} * P_{r}=P_{r}^{3}=\mathcal{R}\left(\frac{1}{1-3 r t}, \frac{t}{1-3 r t}\right)
\end{array}
$$

and by proceeding by mathematical induction we can prove the more general result:

$$
P_{r}^{s}=\mathcal{R}\left(\frac{1}{1-s r t}, \quad \frac{t}{1-s r t}\right)
$$

In classical applications, $s \in N$ and $P_{r}^{s}$ corresponds to a standard product among matrices.

However, let us suppose that $r s=1$ with $r, s \in Q$; then

$$
P_{r}^{s}=P=\mathcal{R}\left(\frac{1}{1-t}, \frac{t}{1-t}\right)
$$

and

$$
P_{1 / s}=\mathcal{R}\left(\frac{1}{1-\frac{t}{s}}, \quad \frac{t}{1-\frac{t}{s}}\right)=\sqrt[s]{P}
$$

in particular,

$$
P_{1 / 2}=\mathcal{R}\left(\frac{1}{1-\frac{t}{2}}, \quad \frac{t}{1-\frac{t}{2}}\right)
$$

is the square root of the Pascal triangle since $P_{1 / 2} * P_{1 / 2}=P$, as can be verified for the first 6 rows by performing the following product:

$$
\left[\begin{array}{cccccc}
1 & 0 & 0 & 0 & 0 & 0 \\
\frac{1}{2} & 1 & 0 & 0 & 0 & 0 \\
\frac{1}{4} & 1 & 1 & 0 & 0 & 0 \\
\frac{1}{8} & \frac{3}{4} & \frac{3}{2} & 1 & 0 & 0 \\
\frac{1}{16} & \frac{1}{2} & \frac{3}{2} & 2 & 1 & 0 \\
\frac{1}{32} & \frac{5}{16} & \frac{5}{4} & \frac{5}{2} & \frac{5}{2} & 1
\end{array}\right] \times\left[\begin{array}{cccccc}
1 & 0 & 0 & 0 & 0 & 0 \\
\frac{1}{2} & 1 & 0 & 0 & 0 & 0 \\
\frac{1}{4} & 1 & 1 & 0 & 0 & 0 \\
\frac{1}{8} & \frac{3}{4} & \frac{3}{2} & 1 & 0 & 0 \\
\frac{1}{16} & \frac{1}{2} & \frac{3}{2} & 2 & 1 & 0 \\
\frac{1}{32} & \frac{5}{16} & \frac{5}{4} & \frac{5}{2} & \frac{5}{2} & 1
\end{array}\right]=\left[\begin{array}{cccccc}
1 & 0 & 0 & 0 & 0 & 0 \\
1 & 1 & 0 & 0 & 0 & 0 \\
1 & 2 & 1 & 0 & 0 & 0 \\
1 & 3 & 3 & 1 & 0 & 0 \\
1 & 4 & 6 & 4 & 1 & 0 \\
1 & 5 & 10 & 10 & 5 & 1
\end{array}\right]
$$


Now, let us consider the Bell matrix $H=\mathcal{R}(h(t) / t, h(t))$ and consider the product of $H$ by itself:

$$
H * H=\mathcal{R}(h(t) / t, \quad h(t)) * \mathcal{R}(h(t) / t, \quad h(t))=\mathcal{R}(h(h(t)) / t, h(h(t)))=\mathcal{R}(f(t) / t, f(t))
$$

therefore $H$ can be seen as the square root of $\mathcal{R}(f(t) / t, f(t))$.

Given $h(t)$ we can compute $f(t)$ : the question is, given $f(t)$, is it possible to compute $h(t)$ such that $f(t)=h(h(t))$ ? We point out that in these computations we are considering formal power series over $\mathbb{R}$ and that we need to have $h(0)=f(0)=0$.

Obviously the problem can be generalized and one can ask the same question for the product of $H$ by itself three times (or more) looking for a function $h(t)$ such that $g(t)=h(h(h(t)))$ for a given $g(t)$ :

$$
\begin{aligned}
H * H * H & =\mathcal{R}(h(t) / t, h(t)) * \mathcal{R}(h(t) / t, h(t)) * \mathcal{R}(h(t) / t, h(t)) \\
& =\mathcal{R}(h(t) / t, h(t)) * \mathcal{R}(h(t) / t, h(t))=\mathcal{R}(h(h(t)) / t, h(h(t))) \\
& =\mathcal{R}(h(h(h(t))) / t, h(h(h(t))))=\mathcal{R}(g(t) / t, g(t)) .
\end{aligned}
$$

Studying fractional iteration of power series is a classical topic, addressed in several classical books such as $[8,14,20]$ and the compositional square root is a particular case. However, as far as we know, this is the first attempt to study the problem in the context of Riordan arrays. Functions of Riordan arrays chunks, in particular the square root of Riordan arrays chunks, have been recently studied in [9] in terms of generalized Lagrange bases and Hermite polynomials. Here we are interested in studying the problem with a generating function approach and identifying the square root for the Riordan array in its entirety. In the next sections, we analyse the square root case and give some hints for general powers. Moreover, a result of Barry [1] is specialized to Bell matrices to find Riordan involutions associated with Bell matrices, another interesting problem related to powers of Riordan arrays.

\section{The Square Root Case}

We start by giving some example of square root matrices.

\section{Self-composition of the Fibonacci Sequence}

Let us consider the generating function of Fibonacci numbers, $h(t)=t /(1-$ $\left.t-t^{2}\right)=t+t^{2}+2 t^{3}+3 t^{4}+5 t^{5}+8 t^{6}+\cdots$ (sequence A000045 in OEIS), and the corresponding Riordan array:

$$
H=\mathcal{R}\left(\frac{1}{1-t-t^{2}}, \frac{t}{1-t-t^{2}}\right)
$$

Since $h(h(t))=t\left(1-t-t^{2}\right) /\left(1-3 t-t^{2}+3 t^{3}+t^{4}\right)=t+2 t^{2}+6 t^{3}+$ $17 t^{4}+50 t^{5}+147 t^{6}+\cdots$ (sequence A270863 in OEIS), matrix $H$ is the square 
root of the Riordan array

$$
H * H=\mathcal{R}\left(\frac{1-t-t^{2}}{1-3 t-t^{2}+3 t^{3}+t^{4}}, \frac{t\left(1-t-t^{2}\right)}{1-3 t-t^{2}+3 t^{3}+t^{4}}\right)
$$

as can be verified on the first 6 rows of the involved matrices by computing the usual row-by-column product:

$$
\left[\begin{array}{llllll}
1 & 0 & 0 & 0 & 0 & 0 \\
1 & 1 & 0 & 0 & 0 & 0 \\
2 & 2 & 1 & 0 & 0 & 0 \\
3 & 5 & 3 & 1 & 0 & 0 \\
5 & 10 & 9 & 4 & 1 & 0 \\
8 & 20 & 22 & 14 & 5 & 1
\end{array}\right] \times\left[\begin{array}{cccccc}
1 & 0 & 0 & 0 & 0 & 0 \\
1 & 1 & 0 & 0 & 0 & 0 \\
2 & 2 & 1 & 0 & 0 & 0 \\
3 & 5 & 3 & 1 & 0 & 0 \\
5 & 10 & 9 & 4 & 1 & 0 \\
8 & 20 & 22 & 14 & 5 & 1
\end{array}\right]=\left[\begin{array}{cccccc}
1 & 0 & 0 & 0 & 0 & 0 \\
2 & 1 & 0 & 0 & 0 & 0 \\
6 & 4 & 1 & 0 & 0 & 0 \\
17 & 16 & 6 & 1 & 0 & 0 \\
50 & 58 & 30 & 8 & 1 & 0 \\
147 & 204 & 131 & 48 & 10 & 1
\end{array}\right]
$$

\section{Self-composition of the Catalan Sequence}

Let us consider the shifted generating function of Catalan numbers (sequence A000108 in OEIS $), h(t)=(1-\sqrt{1-4 t}) / 2=t+t^{2}+2 t^{3}+5 t^{4}+14 t^{5}+42 t^{6}+\cdots$ and the corresponding Riordan array:

$$
H=\mathcal{R}\left(\frac{1-\sqrt{1-4 t}}{2 t}, \quad \frac{1-\sqrt{1-4 t}}{2}\right)
$$

Since $h(h(t))=(1-\sqrt{2 \sqrt{1-4 t}-1}) / 2=t+2 t^{2}+6 t^{3}+21 t^{4}+80 t^{5}+322 t^{6}+\cdots$ (sequence A129442 in OEIS), matrix $H$ is s the square root of the Riordan array

$$
H * H=\mathcal{R}\left(\frac{1-\sqrt{2 \sqrt{1-4 t}-1}}{2 t}, \frac{1-\sqrt{2 \sqrt{1-4 t}-1}}{2}\right)
$$

as can be verified on the first 6 rows of the involved matrices by computing the usual row-by-column product:

$$
\left[\begin{array}{cccccc}
1 & 0 & 0 & 0 & 0 & 0 \\
1 & 1 & 0 & 0 & 0 & 0 \\
2 & 2 & 1 & 0 & 0 & 0 \\
5 & 5 & 3 & 1 & 0 & 0 \\
14 & 14 & 9 & 4 & 1 & 0 \\
42 & 42 & 28 & 14 & 5 & 1
\end{array}\right] \times\left[\begin{array}{cccccc}
1 & 0 & 0 & 0 & 0 & 0 \\
1 & 1 & 0 & 0 & 0 & 0 \\
2 & 2 & 1 & 0 & 0 & 0 \\
5 & 5 & 3 & 1 & 0 & 0 \\
14 & 14 & 9 & 4 & 1 & 0 \\
42 & 42 & 28 & 14 & 5 & 1
\end{array}\right]=\left[\begin{array}{cccccc}
1 & 0 & 0 & 0 & 0 & 0 \\
2 & 1 & 0 & 0 & 0 & 0 \\
6 & 4 & 1 & 0 & 0 & 0 \\
21 & 16 & 6 & 1 & 0 & 0 \\
80 & 66 & 30 & 8 & 1 & 0 \\
322 & 280 & 143 & 48 & 10 & 1
\end{array}\right]
$$

\section{The General Case}

As already observed, given $h(t)$ computing the self composition $h(h(t))$ is simple and proceeding in this way we can find square roots of many Bell matrices. 
The problem to be investigated is the converse, that is, given $f(t)$ how to compute $h(t)$ such that $h(h(t))=f(t)$. We study the problem by a Riordan array approach, as follows.

We consider the Riordan array $S=\mathcal{R}(1, h(t))$ and denote $s_{n, k}=$ $\left[t^{n}\right] h(t)^{k} ;$ the first 6 rows are

$$
S_{6}=\left[\begin{array}{cccccc}
1 & 0 & 0 & 0 & 0 & 0 \\
0 & h_{1} & 0 & 0 & 0 & 0 \\
0 & h_{2} & h_{1}{ }^{2} & 0 & 0 & 0 \\
0 & h_{3} & 2 h_{1} h_{2} & h_{1}{ }^{3} & 0 & 0 \\
0 & h_{4} & 2 h_{1} h_{3}+h_{2}{ }^{2} & 3 h_{1}{ }^{2} h_{2} & h_{1}{ }^{4} & 0 \\
0 & h_{5} & 2 h_{1} h_{4}+2 h_{2} h_{3} & 3 h_{1}\left(h_{1} h_{3}+h_{2}{ }^{2}\right) & 4 h_{1}{ }^{3} h_{2} & h_{1}{ }^{5}
\end{array}\right]
$$

By Eq. (2), we have the relation

$$
\sum_{k=0}^{n} s_{n, k} h_{k}=\left[t^{n}\right] h(h(t))=\left[t^{n}\right] f(t)
$$

which corresponds to a matrix-vector product, as illustrated by the first 6 involved rows:

$$
\left[\begin{array}{cccccc}
1 & 0 & 0 & 0 & 0 & 0 \\
0 & h_{1} & 0 & 0 & 0 & 0 \\
0 & h_{2} & h_{1}{ }^{2} & 0 & 0 & 0 \\
0 & h_{3} & 2 h_{1} h_{2} & h_{1}{ }^{3} & 0 & 0 \\
0 & h_{4} & 2 h_{1} h_{3}+h_{2}{ }^{2} & 3 h_{1}{ }^{2} h_{2} & h_{1}{ }^{4} & 0 \\
0 & h_{5} & 2 h_{1} h_{4}+2 h_{2} h_{3} & 3 h_{1}\left(h_{1} h_{3}+h_{2}{ }^{2}\right) & 4 h_{1}{ }^{3} h_{2} & h_{1}{ }^{5}
\end{array}\right] \times\left[\begin{array}{c}
0 \\
h_{1} \\
h_{2} \\
h_{3} \\
h_{4} \\
h_{5}
\end{array}\right]=\left[\begin{array}{c}
0 \\
f_{1} \\
f_{2} \\
f_{3} \\
f_{4} \\
f_{5}
\end{array}\right]
$$

The matrix-vector product translates into the following system of equations:

$$
\begin{gathered}
h_{1}^{2}=f_{1} \\
h_{1} h_{2}+h_{1}{ }^{2} h_{2}=f_{2} \\
h_{1} h_{3}+2 h_{1} h_{2}{ }^{2}+h_{1}{ }^{3} h_{3}=f_{3} \\
h_{1} h_{4}+\left(2 h_{1} h_{3}+h_{2}{ }^{2}\right) h_{2}+3 h_{1}{ }^{2} h_{2} h_{3}+h_{1}{ }^{4} h_{4}=f_{4} \\
h_{1} h_{5}+\left(2 h_{1} h_{4}+2 h_{2} h_{3}\right) h_{2}+3 h_{1}\left(h_{1} h_{3}+h_{2}{ }^{2}\right) h_{3}+4 h_{1}{ }^{3} h_{2} h_{4}+h_{1}{ }^{5} h_{5}=f_{5}
\end{gathered}
$$

which can be solved in terms of elements $h_{i}$ since each equation introduces only a new unknown with respect to the previous equation; observe that from the second equation we have $h_{2}=f_{2} /\left(h_{1}\left(1+h_{1}\right)\right)$ which implies that $h_{1} \neq-1$ and $h_{1} \neq 0$, if $f_{2} \neq 0$. 
For simplifying, let us consider the quite common case in combinatorics $f_{1}=1$ and $f_{2} \neq 0$, then, from the first equation we have $h_{1}= \pm 1$ but from the previous observation and the additional condition on $f_{2}$ we need to choose the + sign and therefore we have:

$$
\begin{aligned}
h_{1} & =1 \\
h_{2} & =\frac{1}{2} f_{2} \\
h_{3} & =-\frac{1}{4} f_{2}^{2}+\frac{1}{2} f_{3} \\
h_{4} & =\frac{1}{2} f_{4}-\frac{5}{8} f_{2} f_{3}+\frac{1}{4} f_{2}^{3} \\
h_{5} & =-\frac{3}{4} f_{2} f_{4}+f_{2}^{2} f_{3}-\frac{5}{16} f_{2}^{4}-\frac{3}{8} f_{3}^{2}+\frac{1}{2} f_{5}
\end{aligned}
$$

and so on for the next values. This proves that given $f(t)$ with $f(0)=0$, $f^{\prime}(0)=1$ and $f^{\prime \prime}(0) \neq 0$ we can compute the coefficients of the function $h(t)$ by solving the previous linear system. For example, if $f(t)=t /(1-t)$ all coefficients $f_{i}$ are equal to 1 which gives:

$$
h_{1}=1, h_{2}=\frac{1}{2}, h_{3}=\frac{1}{4}, h_{4}=\frac{1}{8}, h_{5}=\frac{1}{16}, \cdots
$$

as expected and illustrated in Sect. 1. Similarly, by considering sequences $f_{1}=$ $1, f_{2}=2, f_{3}=6, f_{4}=17, f_{5}=50, f_{6}=147 \cdots$ and $f_{1}=1, f_{2}=2, f_{3}=$ $6, f_{4}=21, f_{5}=80, f_{6}=322 \cdots$ we find sequences $h_{1}=1, h_{2}=1, h_{3}=2, h_{4}=$ $3, h_{5}=5, h_{6}=8 \cdots$ and $h_{1}=1, h_{2}=1, h_{3}=2, h_{4}=5, h_{5}=14, h_{6}=42 \cdots$ of Fibonacci and Catalan numbers, as illustrated at the beginning of this section.

Since, in general, a matrix can have several square roots (see, e.g., [7]), the previous result means that a Bell matrix has a unique square root which is a Bell matrix that can be computed by the computational method shown before; this is stated in the following theorem:

Theorem 2.1. Given a Bell matrix $F=\mathcal{R}(f(t) / t, f(t))$ with $f(0)=0, f^{\prime}(0)=$ 1 and $f^{\prime \prime}(0) \neq 0$ then there exist a unique Bell matrix $H=\mathcal{R}(h(t) / t, h(t))$ with $h(0)=0, h^{\prime}(0)=1$ and $h^{\prime \prime}(0) \neq 0$ such that $F=H \times H$, that is, $H=\sqrt{F}$. The coefficients of function $h(t)$ can be computed by using the symbolic approach illustrated before.

We observe that different algorithms for computing the coefficients in the expansion of the $r$ th root of a formal power series $f(t)$ under composition can be found in $[2]$.

Next questions is: is it possible to find a closed form expression $h(t)$ from $f(t)$ ? For example, given $f(t)=1 /(1-t)$, how can we find $h(t)=t /(1-t / 2)$ ? Or, given $f(t)=t\left(1-t-t^{2}\right) /\left(1-3 t-t^{2}+3 t^{3}+t^{4}\right)$, how can we find $h(t)=$ $t /\left(1-t-t^{2}\right)$ ? Finally, given $f(t)=(1-\sqrt{2 \sqrt{1-4 t}-1}) / 2$, how can we find $h(t)=(1-\sqrt{1-4 t}) / 2$ ? Obviously, many other examples could be examined. 
Before we start discussing this problem, let's see some features of Bell matrices and their square root.

Theorem 2.2. Let $F=\mathcal{R}(f(t) / t, f(t))$ be a Bell matrix with $f(0)=0, f^{\prime}(0)=$ 1 and $f^{\prime \prime}(0) \neq 0$ and $H=\mathcal{R}(h(t) / t, h(t))$ with $h(0)=0, h^{\prime}(0)=1$ and $h^{\prime \prime}(0) \neq 0$ its square root; moreover let $A_{f}$ and $A_{h}$ be the $A$-sequences of $F$ and $H$. Then, the following relations hold:

$$
\begin{aligned}
f(t) & =h(h(t)) \quad \text { or } \quad f(\bar{h}(t))=h(t) \\
\bar{f}(t) & =\bar{h}(\bar{h}(t)) \quad \text { or } \quad \bar{f}(h(t))=\bar{h}(t) \\
A_{f}(t) & =A_{h}(t) A_{h}\left(\frac{t}{A_{h}(t)}\right) \quad \text { or } \quad A_{f}(h(t))=\frac{h(t)}{t} A_{h}(t) \\
A_{\bar{f}}(t) & =A_{\bar{h}}(t) A_{\bar{h}}\left(\frac{t}{A_{\bar{h}}(t)}\right) \quad \text { or } \quad A_{\bar{f}}(\bar{h}(t))=\frac{\bar{h}(t)}{t} A_{\bar{h}}(t)
\end{aligned}
$$

For example, for the Pascal case $f(t)=t /(1-t)$ and $A(t)=1+t$, thus we obtain the following relations:

$$
\begin{aligned}
h(t) & =\frac{\bar{h}(t)}{1-\bar{h}(t)} \quad \text { or } \quad \bar{h}(t)=\frac{h(t)}{1+h(t)} \\
1+h(t) & =\frac{h(t)}{t} A_{h}(t) \quad \text { or } \quad 1-\bar{h}(t)=\frac{\bar{h}(t)}{t} A_{\bar{h}}(t)
\end{aligned}
$$

However, these relations are equivalent and they do not allow to find $h(t)$.

\section{Guessing $h(t)$}

In some situations, by looking at the very first coefficients of the function $h(t)$ defined in Theorem 2.1, it is possible to guess the generating function, for example consulting the OEIS [12]. This is surely true for the examples just discussed, since they correspond to well-known sequences. Sometimes this is possible also for less well-known sequences. As an example, let us consider the generating function

$$
\begin{aligned}
& f(t)=\frac{\left(1+a t+b t^{2}\right) t}{1+3 a t+\left(2 a^{2}+3 b^{2}\right) t^{2}+3 a b t^{3}+b^{2} t^{4}}=t-2 a t^{2}+\left(4 a^{2}-2 b\right) t^{3}+ \\
& -a\left(8 a^{2}-9 b\right) t^{4}+\left(16 a^{4}-29 a^{2} b+5 b^{2}\right) t^{5}-a\left(a^{2}-2 b\right)\left(32 a^{2}-17 b\right) t^{5}+O\left(t^{6}\right)
\end{aligned}
$$

By using the relations between the coefficients of $f(t)$ and $h(t)$ we find the first values:

$$
h_{1}=1, h_{2}=-a, h_{3}=a^{2}-b, h_{4}=-a^{3}+2 a b, h_{5}=a^{4}-3 a^{2} b+b^{2},
$$

but looking at them it is not easy to guess the corresponding generating function. However, we can make an hypotheses and imagine that it is a rational function, that is, sequence $\left(h_{n}\right)_{n \in N}$ satisfies a linear recurrence relation with 
constant coefficients. We start by looking for a recurrence relation of the second order:

$$
h_{n+2}=c_{1} h_{n+1}+c_{0} h_{n}
$$

By substituting $n=0$ and $n=1$ we have

$$
-a=c_{1}, \quad a^{2}-b=-a c_{1}+c_{0}
$$

hence $c_{0}=-b$ and $c_{1}=-a$. By transforming the recurrence relation into a generating function by the method of coefficients [11], we find:

$$
\frac{h(t)-h_{0}-h_{1} t}{t^{2}}+a \frac{h(t)-h_{0}}{t}+b h(t)=0
$$

that is

$$
h(t)=\frac{t}{1+a t+b t^{2}} .
$$

Finally, by computing $h(h(t))$ we can verify that it corresponds to $f(t)$. This proves that

$$
H=\mathcal{R}\left(\frac{1}{1+a t+b t^{2}}, \frac{t}{1+a t+b t^{2}}\right)
$$

is the square root of the Riordan array:

$H * H=\mathcal{R}\left(\frac{\left(1+a t+b t^{2}\right)}{1+3 a t+\left(2 a^{2}+3 b^{2}\right) t^{2}+3 a b t^{3}+b^{2} t^{4}}, \frac{\left(1+a t+b t^{2}\right) t}{1+3 a t+\left(2 a^{2}+3 b^{2}\right) t^{2}+3 a b t^{3}+b^{2} t^{4}}\right)$,

thus generalizing the Fibonacci case.

If the check $f(t)=h(h(t))$ had not given the desired result, we could have tried a higher order recurrence, which exists if the function $h(t)$ is rational and is computable with the method of coefficient already mentioned. There is the classical GFUN Maple package [16] that allow you to guess the generating function with a similar approach, even in the algebraic case, that is, when the linear recurrence relation has polynomial coefficients. For example, giving the list $0,1,1,2,5,14,42$ as input to the guessgf function of the package, will return the generating function of Catalan numbers $\frac{1-\sqrt{1-4 t}}{2 t}$. As a more complex example, if we consider the series

$$
\begin{aligned}
h(t)= & t+b t^{2}+\left(b^{2}+c\right) t^{3}+b\left(b^{2}+3 c\right) t^{4}+\left(b^{4}+6 b^{2} c+2 c^{2}\right) t^{5} \\
& +b\left(b^{4}+10 b^{2} c+10 c^{2}\right) t^{6}+O\left(t^{7}\right)
\end{aligned}
$$

and give it as input to the seriestoalgeq function of the package, we obtain the equation:

$$
c t h(t)^{2}+(b t-1) h(t)+t=0
$$

whose solution with $h(0)=0$ and $h^{\prime}(0)=1$ yields the generating function of generalized Motzkin numbers:

$$
h(t)=\frac{1-b t-\sqrt{1-2 b t+\left(b^{2}-4 c\right) t^{2}}}{2 c t} ;
$$


classical Motzkin numbers correspond to $b=c=1$ while $b=0$ and $c=1$ gives the aerated function of Catalan numbers. This is what we would obtain starting with $f(t)=h(h(t))$ and applying the computational method illustrated in the previous section.

As a final example, let us consider again the Catalan triangle, which is one of the most studied Riordan array due to the many combinatorial interpretations of the involved numbers,

$$
\mathcal{R}\left(\frac{1-\sqrt{1-4 t}}{2 t}, \frac{1-\sqrt{1-4 t}}{2}\right)
$$

and look for its square root. The computational method gives for the first 6 rows the following decomposition:

$$
\left[\begin{array}{cccccc}
1 & 0 & 0 & 0 & 0 & 0 \\
\frac{1}{2} & 1 & 0 & 0 & 0 & 0 \\
\frac{3}{4} & 1 & 1 & 0 & 0 & 0 \\
\frac{3}{2} & \frac{7}{4} & \frac{3}{2} & 1 & 0 & 0 \\
\frac{55}{16} & \frac{15}{4} & 3 & 2 & 1 & 0 \\
\frac{545}{64} & \frac{143}{16} & \frac{55}{8} & \frac{9}{2} & \frac{5}{2} & 1
\end{array}\right] \times\left[\begin{array}{cccccc}
1 & 0 & 0 & 0 & 0 & 0 \\
\frac{1}{2} & 1 & 0 & 0 & 0 & 0 \\
\frac{3}{4} & 1 & 1 & 0 & 0 & 0 \\
\frac{3}{2} & \frac{7}{4} & \frac{3}{2} & 1 & 0 & 0 \\
\frac{55}{16} & \frac{15}{4} & 3 & 2 & 1 & 0 \\
\frac{545}{64} & \frac{143}{16} & \frac{55}{8} & \frac{9}{2} & \frac{5}{2} & 1
\end{array}\right]=\left[\begin{array}{cccccc}
1 & 0 & 0 & 0 & 0 & 0 \\
1 & 1 & 0 & 0 & 0 & 0 \\
2 & 2 & 1 & 0 & 0 & 0 \\
5 & 5 & 3 & 1 & 0 & 0 \\
14 & 14 & 9 & 4 & 1 & 0 \\
42 & 42 & 28 & 14 & 5 & 1
\end{array}\right]
$$

However, we did not succeed in finding the closed form or an equation for

$$
h(t)=t+\frac{1}{2} t^{2}+\frac{3}{4} t^{3}+\frac{3}{2} t^{4}+\frac{55}{16} t^{5}+\frac{545}{64} t^{6}+O\left(t^{7}\right)
$$

or, equivalently, for

$$
A_{h}(t)=t+\frac{1}{2} t^{2}+\frac{1}{2} t^{3}+\frac{5}{8} t^{4}+\frac{7}{8} t^{5}+\frac{83}{64} t^{6}+O\left(t^{7}\right),
$$

apart from those corresponding to Theorem 2.2

$$
f(t)=\frac{1-\sqrt{1-4 t}}{2}=h(h(t)), \quad \frac{1}{1-h(t)}=\frac{h(t)}{t} A_{h}(t),
$$

which correspond to $A_{f}(t)=\frac{1}{1-t}$. We will return to this example in the next section.

\section{A Solution Through Schröder's Equation}

The computation of $h(t)$ in Theorem 2.1 seems to be connected to the solution of Schröder's equation [17], a functional equation with one independent variable: given the function $f$ the aim is to find the function $\Psi$ such that

$$
s \Psi(t)=\Psi(f(t)),
$$


for some constant $s \neq 1$. If the origin is a fixed point of $f$, i.e., $f(0)=0$, it follows that $\Psi(0)=0$, and if $\Psi^{\prime}(0) \neq 0$, then $s=f^{\prime}(0)$. The compositional inverse satisfies Poincaré's equation,

$$
\bar{\Psi}(s t)=f(\bar{\Psi}(t)) .
$$

Upon iteration of the functional equation we have:

$$
s^{n} \Psi(t)=\Psi(f(f \cdots(f(t))))=\Psi\left(f_{n}(t)\right)
$$

and

$$
\begin{aligned}
& f_{1}(t)=\bar{\Psi}(s \Psi(t))=f(t) \\
& f_{n}(t)=\bar{\Psi}\left(s^{n} \Psi(t)\right),
\end{aligned}
$$

where $n$ is not necessarily positive or integer. In particular, for the functional square root we have

$f_{1 / 2}(t)=\bar{\Psi}\left(s^{1 / 2} \Psi(t)\right), \quad f_{1 / 2}\left(f_{1 / 2}(t)\right)=\bar{\Psi}\left(s^{1 / 2} \Psi\left(\bar{\Psi}\left(s^{1 / 2} \Psi(t)\right)\right)\right)=f_{1}(t)=f(t)$.

Hence, for computing $f_{1 / 2}(t)$ we need to find the Schröder's auxiliary function $\Psi(t)$, and construct the inverse function $\bar{\Psi}(t)$. For example, as illustrated in [5], if $f(t)=2 t(1+t)$ then Schröder's equation is solved by $\Psi(t)=\frac{1}{2} \ln (1+2 t)$ and $\bar{\Psi}(t)=\frac{1}{2}\left(e^{2 t}-1\right)$ which gives $f_{1 / 2}(t)=\frac{1}{2}\left((1+2 t)^{\sqrt{2}}-1\right)$.

However, as observed in [5], it is crucial to note that in the limit $s \rightarrow 1$, all iterates and inverse iterates lose their distinction and degenerate to the identity map, $f_{0}(t)=t$, and the method fails. For this reason, if $f^{\prime}(0)=1$, as in our case, one augments $f_{1}(t)$ in Schröder's equation to $s f_{1}(t)$, and takes the $s \rightarrow 1$ limit only at the very end of the calculations.

Let us show this approach for the Pascal case, by considering $f(t)=\frac{s t}{1-t}$. We need to find a function $\Psi(t)$ with $\Psi(0)=0$ and $\Psi^{\prime}(0) \neq 0$ such that

$$
s \Psi(t)=\Psi\left(\frac{s t}{1-t}\right),
$$

for some constant $s \neq 1$. By assuming that $\Psi(t)=\sum_{k \geq 1} \Psi_{k} t^{k}$ we have:

$$
s \sum_{k \geq 1} \Psi_{k} t^{k}-\sum_{k \geq 1} \Psi_{k}\left(\frac{s t}{1-t}\right)^{k}=0 .
$$

The coefficients in the development of the previous expression should be equal to zero, and since

$$
\begin{aligned}
& {\left[t^{j}\right]\left(\frac{t^{k-1}}{(1-t)^{k}}\right)=\left[t^{j-k+1}\right](1-t)^{-k}=\left(\begin{array}{c}
-k \\
j-k+1
\end{array}\right)(-1)^{j-k+1}} \\
& \quad=\left(\begin{array}{c}
k+j-k+1-1 \\
j-k+1
\end{array}\right)=\left(\begin{array}{c}
j \\
j-k+1
\end{array}\right)=\left(\begin{array}{c}
j \\
k-1
\end{array}\right),
\end{aligned}
$$


where $\left[t^{j}\right]$ is the coefficient of operator, we have:

$$
\begin{aligned}
s & \sum_{k \geq 1} \Psi_{k} t^{k}-\sum_{k \geq 1} \Psi_{k}\left(\frac{s t}{1-t}\right)^{k}=\sum_{k \geq 1} s \Psi_{k} t^{k}-\sum_{k \geq 1} s^{k} \Psi_{k} \sum_{j \geq 0}\left(\begin{array}{c}
j \\
k-1
\end{array}\right) t^{j+1} \\
& =\sum_{k \geq 0} s \Psi_{k+1} t^{k+1}-\sum_{k \geq 0} s^{k+1} \Psi_{k+1} \sum_{j \geq 0}\left(\begin{array}{l}
j \\
k
\end{array}\right) t^{j+1} \\
& =\sum_{k \geq 0} s \Psi_{k+1} t^{k+1}-\sum_{j \geq 0} \sum_{k \geq 0} s^{k+1} \Psi_{k+1}\left(\begin{array}{l}
j \\
k
\end{array}\right) t^{j+1}=0 .
\end{aligned}
$$

Hence, by extracting the $(n+1)$ th coefficient we have:

$$
s \Psi_{n+1}=\sum_{k=0}^{n} s^{k+1} \Psi_{k+1}\left(\begin{array}{l}
n \\
k
\end{array}\right)
$$

or,

$$
\left(1-s^{n}\right) \Psi_{n+1}=\sum_{k=0}^{n-1} s^{k} \Psi_{k+1}\left(\begin{array}{l}
n \\
k
\end{array}\right) .
$$

By varying $n$, we find:

$$
\Psi_{2}=\frac{\Psi_{1}}{1-s}, \Psi_{3}=\frac{\Psi_{1}}{(1-s)^{2}}, \Psi_{4}=\frac{\Psi_{1}}{(1-s)^{3}}, \cdots
$$

and, more generally, $\Psi_{n}=\frac{\Psi_{1}}{(1-s)^{n-1}}$, hence we have the following theorem, where we used $\Psi_{1}=1$ :

Theorem 4.1. The Schröder's equation $s \Psi(t)=\Psi(f(t))$ with $f(t)=\frac{\text { st }}{1-t}$ has solution

$$
\Psi(t)=\sum_{k \geq 1} \frac{t^{k}}{(1-s)^{k-1}}=\frac{t(1-s)}{1-s-t}, \quad \bar{\Psi}(t)=\frac{t(1-s)}{1-s+t} .
$$

Moreover we have:

$$
f_{1 / 2}(t)=\bar{\Psi}(\sqrt{s} \Psi(t))=\frac{\sqrt{s}(1-s) t}{1-s+\sqrt{s} t-t},
$$

where $f(t)=f_{1 / 2}\left(f_{1 / 2}(t)\right)$.

Finally, the solution corresponding to $s=1$ can be found by computing the following limit:

$$
\begin{aligned}
& \lim _{s \rightarrow 1} \frac{\sqrt{s}(1-s) t}{1-s+\sqrt{s} t-t}=\lim _{s \rightarrow 1} \frac{\sqrt{s}(1-s) t}{(1-s)\left(1-\frac{1-\sqrt{s}}{1-s} t\right)} \\
& \quad=\lim _{s \rightarrow 1} \frac{\sqrt{s} t}{1-\frac{1}{1+\sqrt{s}} t}=\frac{t}{1-\frac{t}{2}},
\end{aligned}
$$


as desired. More generally, for what concerns $f_{1 / r}(t)$ we have:

$$
f_{1 / r}(t)=\bar{\Psi}\left(s^{1 / r} \Psi(t)\right)=\frac{s^{1 / r}(1-s) t}{1-s+s^{1 / r} t-t}
$$

and the $s \rightarrow 1$ limit gives $\frac{t}{1-\frac{t}{r}}$, as expected.

By using this approach, in principle, one could compute $h(t)=f_{1 / 2}(t)$ and find the square root of a Bell matrix explicitly. However, solving the Schröder's equation for a generic function $f(t)$ is not simple: the crucial point, in fact, is to determine the function $\Psi$.

As another example, let us consider $f(t)=\frac{s(1-\sqrt{1-4 t})}{2}$ and the corresponding Schröder's equation:

$$
s \sum_{k \geq 1} \Psi_{k} t^{k}=\sum_{k \geq 1} \Psi_{k} s^{k}\left(\frac{1-\sqrt{1-4 t}}{2}\right)^{k} .
$$

We proceed similarly to the previous example and begin by performing the following extraction, related to the generating function $C(t)$ of Catalan numbers:

$$
\begin{aligned}
{\left[t^{j}\right] C(t) } & =\left[t^{j}\right]\left(\frac{1-\sqrt{1-4 t}}{2 t}\right)^{k}=\left[t^{j}\right]\left[(1+w)^{k} \mid w=t(1+w)^{2}\right] \\
& =\frac{1}{j}\left[t^{j-1}\right] k(1+t)^{k-1}(1+t)^{2 j}=\frac{k}{j}\left[t^{j-1}\right](1+t)^{2 j+k-1} \\
& =\frac{k}{j}\left(\begin{array}{c}
2 j+k-1 \\
j-1
\end{array}\right)=\frac{k}{k+2 j}\left(\begin{array}{c}
k+2 j \\
j
\end{array}\right),
\end{aligned}
$$

where $1+w=C(t)$ and we used Lagrange Inversion Formula (see, e.g, [10]). Therefore we have:

$$
\begin{aligned}
& s \sum_{k \geq 1} \Psi_{k} t^{k}=\sum_{k \geq 1} \Psi_{k} s^{k} t^{k} \sum_{j \geq 1} \frac{k}{k+2 j}\left(\begin{array}{c}
k+2 j \\
j
\end{array}\right) t^{j}, \\
& s \sum_{k \geq 1} \Psi_{k} t^{k}=\sum_{j \geq 1}\left(\sum_{k \geq 1} \Psi_{k} s^{k} \frac{k}{k+2 j}\left(\begin{array}{c}
k+2 j \\
j
\end{array}\right)\right) t^{j+k} .
\end{aligned}
$$

Hence, by extracting the $n$th coefficient, we have:

$$
s \Psi_{n}=\sum_{k \geq 1} s^{k} \Psi_{k} \frac{k}{2 n-k}\left(\begin{array}{c}
2 n-k \\
n-k
\end{array}\right)=\sum_{k=1}^{n} s^{k} \Psi_{k} \frac{k}{2 n-k}\left(\begin{array}{c}
2 n-k \\
n
\end{array}\right) .
$$

We can state this result in the following theorem: 
Theorem 4.2. The coefficients of the auxiliary function $\Psi(t)$ in the Schröder's equation $s \Psi(t)=\Psi(f(t))$ with $f(t)=\frac{s(1-\sqrt{1-4 t})}{2}$ satisfy the following recurrence relation:

$$
\left(1-s^{n-1}\right) \Psi_{n}=\sum_{k=1}^{n-1} s^{k-1} \Psi_{k} \frac{k}{2 n-k}\left(\begin{array}{c}
2 n-k \\
n
\end{array}\right) .
$$

By varying $n$ in Theorem 4.2, we find:

$$
\begin{aligned}
& \Psi_{2}=\frac{\Psi_{1}}{1-s}, \Psi_{3}=\frac{2 \Psi_{1}}{(1+s)(1-s)^{2}}, \Psi_{4}=\frac{\Psi_{1}\left(5+s^{2}\right)}{(1+s)\left(1+s+s^{2}\right)(1-s)^{3}}, \\
& \Psi_{5}=\frac{2 \Psi_{1}\left(7+2 s^{2}+3 s^{3}\right)}{(1+s)^{2}\left(1+s^{2}\right)\left(1+s+s^{2}\right)(1-s)^{4}}, \cdots
\end{aligned}
$$

but a general form for $\Psi_{k}$ does not seem to be simple. However we can write, by setting $\Psi_{1}=1$ :

$$
\begin{aligned}
\Psi(t)= & t+\frac{1}{1-s} t^{2}+\frac{2}{(1+s)(1-s)^{2}} t^{3}+\frac{5+s^{2}}{(1+s)\left(1+s+s^{2}\right)(1-s)^{3}} t^{4} \\
& +\frac{2\left(7+2 s^{2}+3 s^{3}\right)}{(1+s)^{2}\left(1+s^{2}\right)\left(1+s+s^{2}\right)(1-s)^{4}} t^{5}+O\left(t^{6}\right) .
\end{aligned}
$$

By using the Poincaré's Eq. (13) it is not difficult to find the first terms in the development of $\bar{\Psi}(t)$ :

$$
\begin{aligned}
\bar{\Psi}(t)= & t-\frac{1}{1-s} t^{2}-\frac{2}{(1+s)(1-s)^{2}} t^{3}+\frac{s^{2}(1+5 s)}{(1+s)\left(1+s+s^{2}\right)(1-s)^{3}} t^{4}+ \\
& -\frac{2 s^{4}\left(2+3 s+7 s^{2}\right)}{(1+s)^{2}\left(1+s^{2}\right)\left(1+s+s^{2}\right)(1-s)^{4}} t^{5}+O\left(t^{6}\right) .
\end{aligned}
$$

Finally,

$$
\begin{aligned}
f_{1 / 2}(t)= & \bar{\Psi}(\sqrt{s} \Psi(t))=\sqrt{s} t+\frac{\sqrt{s}-s}{1-s} t^{2}+\frac{2\left(\sqrt{s}-s-s^{2}+s^{5 / 2}\right)}{(1+s)(1-s)^{2}} t^{3}+ \\
& +\frac{5 \sqrt{s}-5 s-6 s^{2}+7 s^{5 / 2}-6 s^{3}+6 s^{7 / 2}-2 s^{4}+6 s^{9 / 2}-5 s^{5}}{(1+s)\left(1+s+s^{2}\right)(1-s)^{3}} t^{4}+O\left(t^{5}\right)
\end{aligned}
$$

and, by computing the limit, we have:

$$
\lim _{s \rightarrow 1} f_{1 / 2}(t)=t+\frac{1}{2} t^{2}+\frac{3}{4} t^{3}+\frac{3}{2} t^{4}+\frac{55}{16} t^{5}+O\left(t^{6}\right)
$$

as we already found in the previous section.

The results illustrated in the previous examples can be stated in a more general form, by observing that $\left[t^{n}\right] f(t)^{k}$ corresponds to the generic element of the Riordan array $\mathcal{R}(1, f(t))$ and that $\left[t^{n}\right] \bar{f}(t)^{k}$ corresponds to the generic element of the Riordan array inverse $\mathcal{R}(1, \bar{f}(t))$, as defined in (4); the recurrence relation (15) in the next theorem is obtained by inverting the combinatorial identity corresponding to formula (2): 
Theorem 4.3. The coefficients of the auxiliary function $\Psi(t)$ in the Schröder's equation $s \Psi(t)=\Psi\left(f^{[s]}(t)\right)$ with $f^{[s]}(t)=s f(t), f(0)=0$ and $f^{\prime}(0)=1$, satisfy the following recurrence relations $\left(n \geq 2, \Psi_{1} \neq 0\right)$ :

$$
\begin{aligned}
\left(1-s^{n-1}\right) \Psi_{n} & =\sum_{k=1}^{n-1} s^{k-1} \Psi_{k} f_{n, k}, \\
\left(s^{n-1}-1\right) \Psi_{n} & =\sum_{k=1}^{n-1} \Psi_{k} f_{n, k}^{*},
\end{aligned}
$$

where $f_{n, k}$ and $f_{n, k}^{*}$ are the generic elements of the Riordan arrays $\mathcal{R}(1, f(t))$ and $\mathcal{R}(1, \bar{f}(t))$, respectively. If $h(t)$ is the square root of $f(t)$, that is, $f(t)=$ $h(h(t))$, then we have:

$$
h(t)=\lim _{s \rightarrow 1} \bar{\Psi}(\sqrt{s} \Psi(t)) .
$$

Recurrence relations (14) and (15) are equivalent, but when $\bar{f}(t)$ is simpler than $f(t)$, formula (15) can be more suitable for computation. For example, when $f(t)=\frac{(1-\sqrt{1-4 t})}{2}$ we have $\bar{f}(t)=t(1-t)$ and we immediately find $f_{n, k}^{*}=\left[t^{n-k}\right](1-t)^{k}=\left(\begin{array}{c}n-2 k-1 \\ n-k\end{array}\right)$.

\section{Bell Involutions}

In this last section, we wish to observe that another problem related to the powers of a Riordan array, that has been much studied in the literature (see, e.g., $[1,3,4,13])$, concerns the computation of the involutions in the Riordan group: an involution in the group is a Riordan array $D$ such that $D^{2}=I$. In particular, we specialize to Bell matrices an interesting factorization theorem given in [1, Theorem 15], which allows us to find factorization $D_{1}$ of next theorem; factorization $D_{2}$ can be found by observing that if $D=(d(t), h(t))$ is a Riordan array, then $(-d(t), h(t))=-D$. Both factorizations can be verified by applying formula (3).

Theorem 5.1. For a given Bell matrix, the products:

$$
\begin{aligned}
& \mathcal{R}\left(\frac{h(t)}{t}, h(t)\right) * \mathcal{R}\left(\frac{\bar{h}(-t)}{-t}, \bar{h}(-t)\right)=\mathcal{R}\left(\frac{\bar{h}(-h(t))}{-t}, \bar{h}(-h(t))\right)=D_{1} \\
& \mathcal{R}\left(\frac{h(t)}{t}, h(t)\right) * \mathcal{R}\left(\frac{\bar{h}(-t)}{t}, \bar{h}(-t)\right)=\mathcal{R}\left(\frac{\bar{h}(-h(t))}{t}, \bar{h}(-h(t))\right)=D_{2}
\end{aligned}
$$

are Riordan involutions, that is $D_{1}^{2}=D_{2}^{2}=I=(1, t)$.

Let us apply this theorem to the Bell matrices which correspond to the square roots illustrated in the previous sections. If we put $h(t)=\frac{t}{1-t / 2}$ in 
the theorem, we find two decompositions which are very similar to that corresponding to the square root of Pascal triangle:

$$
\mathcal{R}\left(\frac{1}{1-\frac{t}{2}}, \frac{t}{1-\frac{t}{2}}\right) * \mathcal{R}\left(\frac{ \pm 1}{1-\frac{t}{2}}, \frac{-t}{1-\frac{t}{2}}\right)=\mathcal{R}\left(\frac{ \pm 1}{1-t}, \frac{-t}{1-t}\right) .
$$

On the other hand, if we consider $h(t)=\frac{t}{1-t-t^{2}}$, we have $\bar{h}(t)=\frac{-1-t+\sqrt{1+2 t+5 t^{2}}}{2 t}$ and we obtain the Riordan involutions

$$
\begin{aligned}
& \mathcal{R}\left(\frac{1}{1-t-t^{2}}, \frac{t}{1-t-t^{2}}\right) * \mathcal{R}\left(\frac{1-t-\sqrt{1-2 t+5 t^{2}}}{ \pm 2 t^{2}}, \frac{1-t-\sqrt{1-2 t+5 t^{2}}}{2 t}\right) \\
& \quad=\mathcal{R}\left(\frac{1-2 t-t^{2}-\sqrt{1-4 t+6 t^{2}+4 t^{3}+t^{4}}}{ \pm 2 t^{2}}, \frac{1-2 t-t^{2}-\sqrt{1-4 t+6 t^{2}+4 t^{3}+t^{4}}}{2 t}\right) .
\end{aligned}
$$

The next result is quite general and generalizes the previous example so we state it as a corollary. By considering $h(t)=\frac{t}{1+a t+b t^{2}}$ and $\bar{h}(t)=$ $\frac{1-a t-\sqrt{1-2 a t+\left(a^{2}-4 b\right) t^{2}}}{2 b t}$ and vice versa, $h(t)=\frac{1-a t-\sqrt{1-2 a t+\left(a^{2}-4 b\right) t^{2}}}{2 b t}$ and $\bar{h}(t)=$ $\frac{t}{1+a t+b t^{2}}$ in Theorem 5.1, we have:

Corollary 5.2. The Riordan matrices $\mathcal{R}\left(\frac{g(t)}{ \pm t}, g(t)\right)$ with

$$
g(t)=\frac{-1-2 a t-b t^{2}+\sqrt{1+4 a t-2 b t^{2}+4 a^{2} t^{2}+4 a b t^{3}+b^{2} t^{4}}}{2 b t}
$$

and

$$
g(t)=\frac{t}{2 a t-1}
$$

are Riordan involutions.

Finally, with $h(t)=\frac{1-\sqrt{1-4 t}}{2}$, we have $\bar{h}(t)=t(1-t)$ and the Riordan involution:

$$
\begin{aligned}
\mathcal{R} & \left(\frac{1-\sqrt{1-4 t}}{2 t}, \frac{1-\sqrt{1-4 t}}{2}\right) * \mathcal{R}( \pm(1+t),-t(1+t)) \\
& =\mathcal{R}\left(\frac{-1+t+\sqrt{1-4 t}}{ \pm t},-1+t+\sqrt{1-4 t}\right) .
\end{aligned}
$$

\section{Conclusions}

In this work, the problem of computing the square root of a Bell matrix is addressed. In Sect. 2, the problem is solved with a symbolic approach that in many situations allows us to guess the solution. A method for finding the general solution is given in Theorem 4.3 and involves the well-known Schröder's 
equation. The work also illustrates how it is possible to compute an involution from a Bell matrix.

Funding Open access funding provided by Università degli Studi di Firenze within the CRUI-CARE Agreement.

Open Access. This article is licensed under a Creative Commons Attribution 4.0 International License, which permits use, sharing, adaptation, distribution and reproduction in any medium or format, as long as you give appropriate credit to the original author(s) and the source, provide a link to the Creative Commons licence, and indicate if changes were made. The images or other third party material in this article are included in the article's Creative Commons licence, unless indicated otherwise in a credit line to the material. If material is not included in the article's Creative Commons licence and your intended use is not permitted by statutory regulation or exceeds the permitted use, you will need to obtain permission directly from the copyright holder. To view a copy of this licence, visit http://creativecommons. org/licenses/by/4.0/.

\section{References}

[1] Barry, P.: Chebyshev moments and Riordan involutions (2019). arXiv:1912.11845

[2] Brent, R.P., Traub, J.F.: On the complexity of composition and generalized composition of power series. SIAM J. Comput. 9(1), 54-66 (1980)

[3] Cameron, N.T., Nkwanta, A.: On some (pseudo) involutions in the Riordan group. J. Integer Seq. 8, 16 (2005)

[4] Cheon, G.-S., Kim, H., Shapiro, L.W.: Riordan group involutions. Linear Algebra Appl. 428, 941-952 (2008)

[5] Curtright, T., Zachos, C.: Evolution profiles and functional equations. J. Phys. A Math. Theor. 42(48), 485208 (2009)

[6] He, T.-X., Sprugnoli, R.: Sequence characterization of Riordan arrays. Discrete Math. 309(12), 3962-3974 (2009)

[7] Higham, N.: Functions of Matrices. Theory and Computation. SIAM, Berlin (2008)

[8] Kuczma, M., Choczewski, B., Ger, R.: Iterative Functional Equations. Cambridge University Press, Cambridge (1990)

[9] Merlini, D., Nocentini, M.: Functions and Jordan canonical forms of Riordan matrices. Linear Algebra Appl. 565, 177-207 (2019)

[10] Merlini, D., Sprugnoli, R., Verri, M.C.: Lagrange inversion: when and how. Acta Appl. Math. 94, 233-249 (2006)

[11] Merlini, D., Sprugnoli, R., Verri, M.C.: The Method of Coefficients. Am. Math. Monthly 114(1), 40-57 (2007)

[12] OEIS Foundation Inc.: The on-line encyclopedia of integer sequences. https:// oeis.org/ 
[13] Phulara, D., Shapiro, L.: Constructing pseudo-involutions in the Riordan group. J. Integer Seq. 20, 17 (2017)

[14] Riordan, J.: Introduction to Combinatorial Analysis. Princeton University Press, Princeton (1958)

[15] Rogers, D.G.: Pascal triangles, Catalan numbers and renewal arrays. Discrete Math. 22, 301-310 (1978)

[16] Salvy, B., Zimmermann, P.: GFUN: a Maple package for the manipulation of generating and holonomic functions in one variable. ACM Trans. Math. Softw. 20(2), 163-177 (1994)

[17] Schröder, E.: Ueber iterirte functionen. Math. Ann. 3(2), 296-322 (1870)

[18] Shapiro, L.W., Getu, S., Woan, W.J., Woodson, L.: The Riordan group. Discrete Appl. Math. 34, 229-239 (1991)

[19] Sprugnoli, R.: Riordan arrays and combinatorial sums. Discrete Math. 132, 267290 (1994)

[20] Stanley, R.: Enumerative Combinatorics, vol. 1. Cambridge University Press, Cambridge (1997)

Donatella Merlini

Dipartimento di Statistica, Informatica, Applicazioni

Università di Firenze

Firenze

Italy

e-mail: donatella.merlini@unifi.it

Received: November 5, 2020.

Accepted: February 1, 2021.

Publisher's Note Springer Nature remains neutral with regard to jurisdictional claims in published maps and institutional affiliations. 